\title{
Spine and sacroiliac joints on magnetic resonance imaging in patients with early axial spondyloarthritis: prevalence of lesions and association with clinical and disease activity indices from the Italian group of the SPACE study
}

\author{
M. Lorenzin1, A. Ortolan'1, P. Frallonardo¹, S. Vio², C. Lacognata², F. Oliviero', \\ L. Punzi ${ }^{1}$, R. Ramonda ${ }^{1}$ \\ ${ }^{1}$ Rheumatology Unit, Department of Medicine; ${ }^{2}$ Radiology Unit, University of Padova, Italy
}

\section{SUMMARY}

Our aim was to determine the prevalence of spine and sacroiliac joint (SIJ) lesions on magnetic resonance imaging (MRI) in patients with early axial spondyloarthritis (axSpA) and their correlation with disease activity indices.

Sixty patients with low back pain (LBP) ( $\geq 3$ months, $\leq 2$ years, onset $\leq 45$ years), attending the SpA-clinic of the Unità Operativa Complessa Reumatologia of Padova [SpondyloArthritis-Caught-Early (SPACE) study], were studied following a protocol including physical examination, questionnaires, laboratory tests, X-rays and spine and SIJ MRI. Positive spine and SIJ MRI and X-rays images were scored independently by 2 readers using the SPARCC method, modified Stoke ankylosing spondylitis spine score and New York criteria. The axial pain and localization of MRI-lesions were referred to 4 sites: cervical/thoracic/lumbar spine and SIJ. All patients were classified into three groups: patients with signs of radiographic sacroiliitis (r-axSpA), patients without signs of r-axSpA but with signs of sacroiliitis on MRI (nr-axSpA MRI SIJ+), patients without signs of sacroiliitis on MRI and X-rays (nr-axSpA MRI SIJ-).

The median age at LBP onset was $29.05 \pm 8.38$ years; $51.6 \%$ of patients showed bone marrow edema (BME) in spine-MRI and $56.7 \%$ of patients in SIJ-MRI. Signs of enthesitis were found in $55 \%$ of patients in the thoracic district. Of the $55 \%$ of patients with BME on spine-MRI, $15 \%$ presented presented a negative SIJMRI. There was a significant difference between these cohorts with regard to the prevalence of radiographic sacroiliitis, active sacroiliitis on MRI and SPARCC SIJ score.

The site of pain correlated statistically with BME lesions in thoracic and buttock districts. Since positive spine-MRI images were observed in absence of sacroiliitis, we can hypothesize that this finding could have a diagnostic significance in axSpA suspected axSpA.

Key words: Axial spondyloarthritis; early onset spondyloarthritis; disease activity; clinimetric indices spine; sacroiliac joints.

Reumatismo, 2016; 68 (2): 72-82

\section{INTRODUCTION}

pondyloarthritis (SpA) is a group of chronic inflammatory rheumatic diseases that share overlapping features and that can be divided into two main groups: axial SpA (axSpA) and peripheral SpA (pSpA) (1-3). Thanks to the development of biotechnological drugs, significant progress has been made in the treatment of axSpA making early diagnosis and treatment even more relevant. Rheuma- tologists are making every effort to assess disease activity not only with the intent of initiating treatment as soon as possible but also to monitor patients' response to therapy. In accordance with the revised New York criteria, conventional radiographs of the sacroiliac joint (SIJ) are frequently used to detect sacroiliitis, a typical expression of radiographic axSpA ( $\mathrm{r}-\mathrm{axSpA})$ or of ankylosing spondylitis (AS) (4). This method has however proved to be inadequate to diagnose a patient with suspected 
early axSpA, as it is able to detect only structural damage, which is a characteristic of advanced disease stage $(1,4)$. Magnetic resonance imaging (MRI), which can detect both inflammatory lesions and structural damage of the SIJ, can be used in addition to radiographs when $\mathrm{SpA}$ is suspected (5-8). In patients with earlyonset axSpA without evidence of radiographic sacroiliitis, MRI can, in fact, detect inflammatory lesions before bone damage becomes visible. As it has been seen that these patients respond quickly and effectively to anti-tumor necrosis factor- $\alpha$ drugs $(3,9-11)$, it has become urgent to identify early stages of the disease in order to begin appropriate treatment as early as possible.

The Assessment of SpondyloArthritis International Society (ASAS) has recently established classification criteria for patients with suspected axSpA, including those with and without radiographic sacroiliitis (5). As far as imaging is concerned, the ASAS criteria require the presence of sacroiliitis on a MRI or conventional radiography in addition to at least one of the signs of $\mathrm{SpA}$ for patients with chronic low back pain (LBP) with age at onset $\leq 45$ years. Positive SIJ MRI scans were defined by the ASAS/Outcome Measures in Rheumatology MRI working group (OMERACT) as the presence of inflammatory lesions such as bone marrow edema (BME) which is highly suggestive of $\operatorname{SpA}(8)$.

Whether structural SIJ lesions should be added to this definition and if structural and inflammatory spinal lesions could contribute to detecting ax $\mathrm{SpA}$ remains to be established (12). Inflammatory spinal lesions on MRIs may nevertheless occur in the absence of SIJ involvement (13). Spinal MRIs in AS patients have uncovered abnormalities in this district even before they are noted on plain radiographs (14). The introduction of fat-suppression sequences has allowed the visualization of lesions within bone marrow that may be obscured on MRI by marrow fat. These lesions include BME adjacent to vertebral endplates at the attachment of the annulus fibrosus to the vertebral rim and at the insertion of anterior and posterior longitudinal ligaments, both within the facet joints. Since there is evidence that spondylitis may also occur prior to or even without sacroiliitis, it was considered important to define the characteristics of a spinal MRI considered positive for inflammation.

The ASAS/OMERACT working group thus set out to define spinal MRIs positive for inflammatory lesions (spondylitis) and structural changes (fat deposition) (12). It is also unknown whether the localization of lesions is correlated to the site of axial pain.

Imaging of the thoracic spine, which is often involved in axSpA, has not yet been taken into consideration when structural damage is being evaluated (15-17). The goal of this study is to determine the prevalence of spine and SIJ lesions on MRI and their correlation with disease activity indices in patients with early axSpA included in the SpondyloArthritis Caught Early (SPACE) Italian cohort.

\section{MATERIALS AND METHODS}

\section{Patients}

Patients who were at least 16 years old, suffering from inflammatory LBP $(\geq 3$ months, $\leq 2$ years, onset $<45$ years) of unknown origin and referred to a rheumatologist were included in the Italian section of the SPACE study.

This is an ongoing observational cohort study, which was originally launched at the Leiden University Medical Centre in January 2009. In March 2012 the SpA Study Group of the Rheumatology Unit at the University of Padua was involved in the SPACE study. Eligible patients underwent physical examinations, laboratory tests, SIJ and spinal plain radiographs and MRIs, following a standardized protocol. The patients also completed questionnaires on disease activity, physical functioning, pain, and disease-related impairment. Axial pain and MRI lesions were localized in 4 sites: in the cervical/thoracic/lumbar spine and the SIJ. An experienced rheumatologist made the diagnosis of axSpA. 
In order to meet the ASAS criteria (5), it was necessary to verify if the patients had MRI evidence of active inflammatory lesions of the SIJ with definite BME, which is highly suggestive of sacroiliitis. After the X-rays and MRI images were read, the patients were divided into three cohorts: those with early signs of r-axSpA, those without signs of r-axSpA but with signs of active sacroiliitis on MRI (nraxSpA MRI SIJ+), those without signs of sacroiliitis on MRI and plain radiograph (nr-axSpA MRI SIJ-). Only baseline (t0) data were used in these analyses. At that time point, all the patients were being treated with non-steroidal anti-inflammatory drugs. No patients were treated with synthetic or biological disease modifying anti-rheumatic drugs. The local medical ethical committee approved the study and informed consent was obtained from all patients at study inclusion.

\section{Magnetic resonance imaging assessments} SIJ and spinal MRIs were performed at baseline using a $1.5 \mathrm{~T}$ scanner Magnetom Harmony (Siemens AG Medical Solutions, Munich, Germany) with phased-array surphace coil, acquiring T1-weighted turbo spin echo (T1TSE; TR 550/TE 10) and short-tau inversion recovery (STIR; TR 2500/TE60) sequences.

The coronal oblique and sagittal views of the SIJ and spine were in $4 \mathrm{~mm}$ slice thicknesses.

The images were analyzed independently by two expert radiologists trained in scoring MRIs in accordance with the ASAS definition and the SPARCC scoring system $(18,19)$. If the two readers scored positive, the image was scored accordingly. All readers were blinded for clinical and laboratory data, and for the results of the other imaging methods.

The mean scores were calculated using those of both of the readers. It was performed intra and inter-observer reliability. All of the inflammatory lesions typical of SpA were graded using the SPARCC scoring system: the SIJ is graded positive if $\geq 1$ BME lesion highly suggestive of $\mathrm{SpA}$ is visible on $\geq 2$ consecutive slices or if several BME lesions highly suggestive of SpA are visible on a single slice (8).

The presence of only synovitis, enthesitis, or capsulitis without BME is not sufficient for a positive reading.

According to the SPARCC scoring method, the presence of an increased signal corresponding to BME lesions on SIJMRIs should be noted on 6 consecutive coronal slices selected as representing the synovial compartment of the SIJ.

The left and right SIJ MRIs were divided into quadrants for a total of 8 per coronal slice. Each quadrant was assessed and evaluated for the presence (scored 1) or absence (scored 0) of BME. To each coronal slice per SIJ was given an additional score of 1 for the presence of an intense signal and an additional score of 1 for a deep lesion, defined as a homogeneous, unequivocal increase in a signal $1 \mathrm{~cm}$ from the articular surface.

The maximum possible score across 6 slices was 48 for the presence of BME, 12 for intense edema, and 12 for deep edema, for a maximum possible total score of 72 (18). Structural lesions on SIJ-MRIs were also evaluated. According to the ASAS/ OMERACT MRI group, BME and fatty lesions on spinal MRIs are considered when they are visible on $\geq 2$ consecutive slices, while the presence of $\geq 1$ slice is enough for structural lesions (erosions, sindesmophytes) (12). For the spine, the 6 most severely affected disco-vertebral units (DVUs) were selected and each was divided into 4 quadrants, with each quadrant assessed for the presence (scored 1) or absence (scored 0) of BME. Each quadrant was scored on 3 consecutive sagittal slices per DVU, yielding a maximum possible score of 12 per DVU for BME. Each sagittal slice per DVU was given an additional score of 1 for the presence of an intense signal and an additional score of 1 for a deep lesion, defined as a homogeneous, unequivocal increase in STIR signal $>1 \mathrm{~cm}$ from the vertebral end plate. The maximum possible score for all 6 DVUs was 72 for the presence of BME, 18 for intense edema, and 18 for deep edema, for a maximum possible total score of 
108 (19). The structural lesions on spinal MRIs were also evaluated.

\section{Radiographs assessments}

Lateral view radiographs of the cervical and lumbar spine and anterior-posterior view radiographs of the pelvis were taken. The images were obtained with a Philips vertical bucky, with a focus-film distance of $140 \mathrm{~cm}$, film size of $18 \times 43 \mathrm{~cm}$. The images were read independently by two expert trained musculoskeletal radiologists, blinded for patients' characteristics, clinical outcome and for the results of the other imaging methods. The mean scores were calculated using those of both of the readers. It was performed intra and interobserver reliability.

The modified Stoke ankylosing spondylitis spine score (mSASSS) scoring method modified by Creemers was used (16). According to this method, lateral views of the anterior vertebral corners (VCs) of the cervical (lower border of $\mathrm{C} 2$ to upper border of T1) and lumbar (lower border of T12 to upper border of S1) segments (a total of $24 \mathrm{VCs}$ ) are scored for the presence of erosions and/or sclerosis and/or squaring ( 1 point), syndesmophytes ( 2 points) and bridging syndesmophytes (3 points). The total score ranges from 0 to 72 (16). Evaluation of the SIJ was based on the New York criteria (4), with scores ranging from 0 to $4(0=$ no change, $1=$ look slightly faded edge joint, pseudo-widening or narrowing of the rhyme, mild subchondral sclerosis, 2=irregular margin joint with images of erosion, shrinkage of rhyme, subchondral sclerosis evident, $3=$ erosions and subchondral sclerosis evident with initial synostosis, 4=complete ankylosis).

\section{Clinical evaluation, questionnaires and laboratory tests}

The clinical evaluation focused on an examination of the spine, SIJ and entheses, using the Bath ankylosing spondylitis metrology index (BASMI) and the Maastricht ankylosing spondylitis enthesitis score (MASES) as a guide. The patients' disease activity and physical functioning were assessed using self-reported ques- tionnaires and composite indices: the Bath ankylosing spondylitis disease activity index (BASDAI), the Bath ankylosing spondylitis functional index (BASFI), the ankylosing spondylitis disease activity score (ASDAS), the visual analogue scale (VAS pain), the VAS night pain scale, the VAS disease activity, the Bath ankylosing spondylitis patient global score (BASG1), the BASG2, the health assessment questionnaire (HAQ).

Patients' erythrocyte sedimentation rate (ESR) (normal range $0-15 \mathrm{~mm} / \mathrm{h}$ ) and high sensitivity $\mathrm{C}$ reactive protein (hsCRP) (normal range 0-6 mg/L) were also evaluated.

\section{Statistical analyses}

Odds ratio (OR) was used to assess the association at $\mathrm{t} 0$ between the site of axial pain and the localization of the inflammatory and structural lesions on the spinal and SIJ MRIs. Cohen's Kappa was used for intra and inter-observer reliability. The Kruskal Wallis (ANOVA) was used to compare at t0 the following indices: clinical (BASMI, MASES), serological (ESR, hsCRP), functional (BASFI, HAQ, BASG1, BASG2, VAS pain, VAS night pain, VAS disease activity), disease activity (BASDAI, ASDAS) indices and imaging score (mSASSS, NY score, SPARCC SIJ and SPARCC 6-DVU). A P value $<0.05$ was considered significant.

\section{RESULTS}

One hundred percent of the 60 patients studied fulfilled the ASAS criteria for axSpA; $23(38.3 \%)$ patients were diagnosed as having r-axSpA, $17(28.3 \%)$ as having nr-axSpA MRI SIJ+ and 20 (33.3\%) as having nr-axSpA MRI SIJ-.

The median age at LBP onset was $29.05 \pm 8.38$ years, $45 \%$ were male, $38.3 \%$ of the patients were HLA-B27+; there was axial involvement in $34(55.7 \%)$ patients and axial/peripheral involvement in $27(44.3 \%)$ patients. High prevalence of psoriasis and heel enthesitis (respectively $34.4 \%$ and $80.3 \%$ ) were noted. Other patient characteristics and SpA features are outlined in Table I. 
Table I - Baseline characteristics of the 60 patients studied [Italian section of the SpondyloArthritis Caught-Early (SPACE) study].

\begin{tabular}{|c|c|}
\hline Age of onset back pain, mean ( \pm SD) & $29.05( \pm 8.38)$ \\
\hline Male, $n(\%)$ & $26(43.3 \%)$ \\
\hline Duration (months) back pain, mean $( \pm S D)$ & $12.62( \pm 5.85)$ \\
\hline Only axial involvement, $\mathrm{n}(\%)$ & $34(56.7 \%)$ \\
\hline Axial and peripheral involvement, $\mathrm{n}(\%)$ & $27(43.3 \%)$ \\
\hline HLA-B27 positive, $n(\%)$ & $22(36.7 \%)$ \\
\hline Positive family history of SpA, $n(\%)$ & $29(48.3 \%)$ \\
\hline IBP, n (\%) & $60(100 \%)$ \\
\hline Peripheral arthritis, $n(\%)$ & $26(43.3 \%)$ \\
\hline Psoriasis, n (\%) & $21(35 \%)$ \\
\hline Dactylitis, n (\%) & $15(25 \%)$ \\
\hline Heel enthesitis, $n(\%)$ & $48(80 \%)$ \\
\hline Uveitis, n (\%) & $4(6.7 \%)$ \\
\hline IBD, n (\%) & $8(13.3 \%)$ \\
\hline Preceding infection, $\mathrm{n}(\%)^{\star}$ & $2(3.3 \%)$ \\
\hline Good response to NSAIDs, $n$ (\%) & $58(96.7 \%)$ \\
\hline Elevated CRP/ESR, $n$ (\%) & $33(55 \%)$ \\
\hline Cervical pain, $n(\%)$ & $39(65 \%)$ \\
\hline Thoracic pain, $n(\%)$ & $28(46.6 \%)$ \\
\hline Buttock pain, $n(\%)$ & $49(81.6 \%)$ \\
\hline Alternating buttock pain, $\mathrm{n}(\%)$ & $26(43.3 \%)$ \\
\hline Morning stiffness, $\mathrm{n}(\%)$ & $57(95 \%)$ \\
\hline Night pain, $n(\%)$ & $58(96.7 \%)$ \\
\hline Sacroilitis MRI, $\mathrm{n}(\%)^{\circ}$ & $32(53.3 \%)$ \\
\hline Sacroiliitis X-ray, $n(\%) \#$ & $23(38.3 \%)$ \\
\hline
\end{tabular}

SD, standard deviation; HLA-B27, human leukocyte antigen; SpA, spondyloarthritis; IBP, inflammatory back pain; IBD, inflammatory bowel disease; NSAID, non-steroidal anti-inflammatory drugs; CRP, C-reactive protein; ESR, erythrocyte sedimentation rate; MRI, magnetic resonance imaging. *Balanitis, urethritis or cervicitis; 'sacroilitis MRI according the Assessment of SpondyloArthritis International Society/European League Against Rheumatism (ASAS/EULAR); \#Sacroiliitis X-ray according New York criteria.

Of the studied patients, $65 \%, 46.6 \%$, $100 \%$, and $81.6 \%$, respectively, complained about cervical/thoracic/lumbar/ buttock pain.

All spine and SIJ on MRI images were evaluated by two expert radiologists and the inter-observer reliability was respectively good to moderate (kappa 0.73 for inflammatory lesions and 0.58 for structural lesions on spine MRI) and good to moderate (kappa 0.78 for inflammatory lesions and 0.60 for structural lesions on SIJ MRI). The inter-observer reliability for all X-rays images was good (kappa
0.79 for spine radiological lesions and kappa 0.77 for SIJ radiological lesions). The intra-observer reliability was moderate to good for all spine and SIJ images on X-rays and MRI (mean kappa-coefficients between 0.59 and 0.80 for SIJ). Forty-two patients (70\%) presented structural and/or inflammatory lesions on SIJMRIs at t0 (on the right SIJ in $60 \%$ of the patients and on left one in $53.3 \%$ of the patients). BME lesions were present in $34(56.7 \%)$ patients $(50 \%$ on the right SIJ and $38.33 \%$ on the left one). Structural lesions on SIJ-MRI were present in 
Table II - The prevalence of inflammatory and structural lesions at baseline in three cohorts.

\begin{tabular}{|l|l|l|l|}
\hline & r-axSpA & nr-axSpA MRI SIJ+ & nr-axSpA MRI SIJ- \\
\hline Total number of patients & $23(38.3 \%)$ & $17(28.3 \%)$ & $20(33.3 \%)$ \\
\hline SIJ total lesions on MRI & $22(95.6 \%)$ & $17(100 \%)$ & $3(15 \%)$ \\
\hline BME lesions & $18(81.7 \%)$ & $17(100 \%)$ & $0(0 \%)$ \\
\hline Sclerosis lesions & $13(56.5 \%)$ & $2(11.8 \%)$ & $3(15 \%)$ \\
\hline Fatty lesions & $5(21.7 \%)$ & $2(11.8 \%)$ & $1(5 \%)$ \\
\hline Erosive lesions & $5(21.7 \%)$ & $1(5.9 \%)$ & $0(0 \%)$ \\
\hline Spine total lesions on MRI & $19(82.6 \%)$ & $14(82.3 \%)$ & $10(50 \%)$ \\
\hline BME lesions & $16(69.6 \%)$ & $10(58.8 \%)$ & $6(30 \%)$ \\
\hline Enthesitis lesions & $12(52.2 \%)$ & $8(47.1 \%)$ & $5(25 \%)$ \\
\hline Fatty lesions & $6(26.1 \%)$ & $4(23.5 \%)$ & $3(15 \%)$ \\
\hline Sclerosis/syndesmophytes lesions & $6(26.1 \%)$ & $4(23.5 \%)$ & $4(20 \%)$ \\
\hline Erosive lesions & $1(4.3 \%)$ & $2(11.8 \%)$ & $2(10 \%)$ \\
\hline
\end{tabular}

r-axSpA, patients with signs of radiographic sacroiliitis; nr-axSpA MRI SIJ+, patients with no signs of radiographic sacroiliitis but with signs of sacroiliitis on magnetic resonance imaging; nr-axSpA MRI SIJ-, subjects with no signs of sacroiliitis neither on MRI not on X-rays; SIJ, sacroiliac joints; BME, bone marrow edema.

$22(36.7 \%)$ patients $(26.7 \%$ on the right SIJ and $30 \%$ on the left one). Thirty-three $(55 \%)$ patients presented inflammatory and/or structural lesions on the spinal MRI at t0. BME lesions at the anterior corner of the spine were present in $51.6 \%$ $(18.3,30$ and $31.7 \%$, respectively, in the cervical/thoracic/lumbar regions). Structural spine lesions were present in $35 \%$ $(17,13,17 \%$, respectively, in the cervi$\mathrm{cal} /$ thoracic/lumbar regions). Signs of enthesitis were found in $35(58.3 \%)$ patients: at the level of the cervical spine in $5 \%$ of the patients, of the thoracic spine in $55 \%$ of the patients, at the lumbar spine in $6 \%$ of the patients.

The $9(15 \%)$ patients with inflammatory lesions on spinal MRIs showed no abnormalities on the SIJ ones, while $12(20 \%)$ patients without active sacroiliitis on SIJ MRIs did not presented lesions on the spinal MRIs.

The prevalence of inflammatory and structural lesions at t0 in the three cohorts (r-axSpA, nr-axSpA MRI SIJ + and nraxSpA MRI SIJ) are outlined in Table II. An increased prevalence of structural lesions on SIJ MRI that was found in the r-axSpA patients with respect to the other two cohorts would support the presence of sacroiliitis involvement on standard X- rays. R-axSpA and nr-axSpA MRI SIJ+ patients had more inflammatory and structural spinal lesions with respect to the nraxSpA MRI SIJ-patients (Table II).

The OR between the site of pain and the localization of BME lesions was, respectively, 20.78 [confidence interval (CI):0.39-11.05; $\mathrm{P}=$ not significant (NS)], 163.93 (CI:3.31-81.28; $\mathrm{P}=0.0006), 0.34$ (CI:0.01-17.91; $\mathrm{P}=\mathrm{NS}$ ) for the cervical/ thoracic/lumbar spine areas and 304.88 (CI: 1.71-546.56; $\mathrm{P}=0.0203$ ) for the buttocks.

The association of pain with structural lesions on the MRIs of the same site was not significant except for the buttock pain/ structural SIJ MRI lesions (OR=70.1; CI: 0.84-58.40; $\mathrm{P}=0.0122$ ). The association between thoracic pain and enthesitis of the thoracic district was found to be significant ( $\mathrm{OR}=32.69$; CI: 1.096-9.748; $\mathrm{P}=0.0336$ ). Clinical and disease activity indices and imaging scores at t0 in the three cohorts are outlined in Table III.

The ANOVA test uncovered a significant difference in the prevalence of radiographic sacroiliitis, active sacroiliitis on MRI and the SPARCC SIJ score in the three cohorts. No differences in the clinical and disease activity indices between the groups were found. 
Table III - Clinical and disease activity indices and imaging scores at baseline in the 60 patients studied and in the three cohorts.

\begin{tabular}{|c|c|c|c|c|c|}
\hline $\begin{array}{l}\text { Disease activity indices } \\
\text { and imaging scores }\end{array}$ & $\begin{array}{l}\text { Cohort } 1 \\
\text { r-axSpA, } n=23\end{array}$ & $\begin{array}{l}\text { Cohort } 2 \text { nr-axSpA MRI } \\
\text { SIJ+, n=17 }\end{array}$ & $\begin{array}{l}\text { Cohort } 3 \text { nr-axSpA } \\
\text { MRI SIJ-, n=20 }\end{array}$ & $P^{*}$ & Total $=60 \mathrm{pts}$ \\
\hline $\mathrm{ESR}(\mathrm{mm} / \mathrm{h})$, mean $(\mathrm{SD})$ & $17.57(14.45)$ & $18.41(12.75)$ & $18.95(21.83)$ & 10 & $18.27(16.61)$ \\
\hline hsCRP (mg/L), mean (SD) & $2.54(2.79)$ & $1.56(1.71)$ & $2.76(3.76)$ & ns & $2.11(2.50)$ \\
\hline HLA B27, n(\%) & $11(47.8)$ & $9(52.9 \%)$ & $3(15 \%)$ & ns & $23(38.3)$ \\
\hline BASMI, mean (SD) & $0.74(1.01)$ & $1.06(1.35)$ & $0.75(0.97)$ & ns & $1.83(1.09)$ \\
\hline MASES, mean (SD) & $2.87(2.39)$ & $4.35(2.69)$ & $3.6(2.37)$ & ns & $3.51(2.51)$ \\
\hline BASFI, mean (SD) & $16.22(23.96)$ & 24.74(23.19) & $15(15.73)$ & ns & $18.23(21.37)$ \\
\hline $\mathrm{HAQ}$, mean (SD) & $0.32(0.52)$ & $0.48(0.61)$ & $0.38(0.35)$ & ns & $0.38(0.49)$ \\
\hline BASG1, mean (SD) & $3.26(2.93)$ & $4.24(2.73)$ & $3.85(3.36)$ & ns & $3.73(3.01)$ \\
\hline BASG2, mean (SD) & $4.78(2.78)$ & $5.47(2.79)$ & $5.05(2.98)$ & ns & $5.07(2.82)$ \\
\hline VAS pain, mean (SD) & $3.70(3.01)$ & $4.06(3.07)$ & $4.55(3.27)$ & ns & $4.08(3.08)$ \\
\hline VAS disease activity, mean (SD) & $3.39(2.86)$ & $4.36(3.30)$ & $4.5(3.55)$ & ns & $4.03(3.21)$ \\
\hline VAS pain night, mean (SD) & $3.61(3.63)$ & $3.71(3.57)$ & $3.8(3.44)$ & ns & $3.07(3.49)$ \\
\hline BASDAI, mean (SD) & $38.02(25.58)$ & $53(25.61)$ & $46.38(23.75)$ & ns & $45.05(25.33)$ \\
\hline ASDAS, mean (SD) & $2.45(0.79)$ & $2.7(0.86)$ & $2.50(0.87)$ & ns & 2.53(0.81) \\
\hline Sacroiliitis X-ray, ${ }^{\circ} \mathrm{n}(\%)$ & $23(100 \%)$ & $0(0)$ & $0(0)$ & $<0.001$ & $25(41.7)$ \\
\hline Sacroililitis MRI,\# n (\%) & $17(73.9 \%)$ & $17(100 \%)$ & $0(0)$ & $<0.001$ & $32(53.3)$ \\
\hline mSASSS, mean (SD) & $3.26(3.21)$ & $3.53(4.86)$ & $3(3.73)$ & ns & $3.25(3.84)$ \\
\hline Score SIJ, mean (SD) & $1.30(0.63)$ & $0(0)$ & $0(0)$ & ns & $0.5(0.75)$ \\
\hline SPARCC spine, mean (SD) & $7.26(12.33)$ & $5.18(15.52)$ & $2.05(3.49)$ & ns & $4.93(11.44)$ \\
\hline SPARCC SIJ, mean (SD) & $15.35(16.57)$ & $12.53(9.15)$ & $0(0)$ & $<0.001$ & $9.43(13.10)$ \\
\hline
\end{tabular}

r-axSpA, patients with signs of radiographic sacroiliitis; nr-axSpA MRI SIJ+, patients without signs of r-axSpA but with signs of sacroiliitis on magnetic resonance imaging; nr-axSpA MRI SIJ-, patients without signs of sacroiliitis on MRI and X-rays; ESR, erythrocyte sedimentation rate; SD, standard deviation; hsCRP, high sensitive C-reactive protein; ns, not significant; HLA-B27, human leukocyte antigen; BASMI, bath ankylosing score metrology index; MASES, Maastricht ankylosing spondilities enthesitis score; BASFI, bath ankylosing spondylitis functional index; HAQ, health assessment questionnaire; BASG1, bath ankylosing spondylitis patient global score 1; BASG2, bath ankylosing spondylitis patient global score 2; VAS, visual analogue scale; BASDAI, bath ankylosing spondylitis disease activity index; ASDAS, ankylosing spondylitis disease activity score; mSASSS, modified stoke ankylosing spondylitis spine score; SIJ, sacroiliac joints; SPARCC, Spondyloarthritis Research Consortium of Canada. *P analysis of variance (Kruskal-Wallis) a t0: $\mathrm{P}<0.05$; 'sacroiliitis X-ray according New York criteria; \#sacroiilitis MRI according the Assessment of SpondyloArthritis International Society/European League Against Rheumatism (ASAS/EULAR).

\section{DISCUSSION}

Modern imaging technology of the pelvis and spine has become a crucial tool for diagnosing, classifying and monitoring axSpA. The traditional radiograph is currently used in clinical practice to evaluate structural bone changes which are an expression of advanced pathological processes such as sacroiliitis in AS. Radiographic sacroiliitis, which was included in the New York criteria (1984) (4), has long been taken into consideration. Over the past two decades, with the advent and development of new imaging techniques, efforts have been made to diagnose AS and
axSpA at ever-earlier stages before structural damage has occurred in order to enhance treatment efficacy $(1,3,5,7)$. MRI, which can detect inflammatory lesions and signs of active processes even before structural bone damage has occurred, is widely used in clinical practice to evaluate patients with LBP and suspected axSpA $(8,12)$. A positive MRI of the SIJ has recently been included in the ASAS classification criteria, whose definition was based exclusively on the presence of inflammation of SIJ $(5,7,8,20)$. Some studies (12, $21,22)$ have recently taken into consideration structural and inflammatory lesions that can be seen on spinal MRIs. The type 
of injury most frequently observed in ax$\mathrm{SpA}$ is BME of the anterior vertebral corners, which is an expression of anterior osteitis (19). Another type of lesion that is detected is the replacement of vertebral angles with adipose tissue (fatty lesions), which seems to be less specific and occur later in $\operatorname{SpA}(23,24)$. The presence of $\mathrm{BME}$ at the posterior vertebral corners appears to be highly specific for this disease, but its use as a diagnostic criterion has been limited by low sensitivity. Just as the ASAS/OMERACT MRI study group (8, $12)$, we analyzed the prevalence and type of inflammatory and structural lesions in a cohort of patients with early stage axSpA. The prevalence of BME lesions in MRIs of the SJI was high in our study, but it was even higher than the prevalence of structural lesions, thus underlining the peculiarity of this method in the visualization of inflammatory findings in comparison to morphological abnormalities. A significant prevalence of BME lesions in the anterior vertebral corners was also observed on MRIs of the spine. Consistent with results reported in previous studies (23-25), the finding highlights the importance of the involvement of the spine from the very first phase of the inflammatory process in axSpA. A high prevalence of other inflammatory signs on MRI linked to enthesitis especially in the thoracic spine were found in our patients suggesting involvement of this district in the early stages of axSpA. From the data reported in the literature $(8,12)$, it is unclear whether the location of lesions on MRI is also associated to the site of axial pain. A significant association between the site of pain and BME lesions in the thoracic and buttock district was noted in our patients. This result seems to indicate that the location of axial pain (thoracic and buttock pain) could be used as a specific predictor of the presence of inflammatory lesions on MRI. The association between the site of pain and the localization of MRI lesions is less striking with regard to structural damage, which was significant only for buttock pain and structural lesions on MRIs of the SIJ. Several studies $(12,13,26,27)$ have systematically evaluated the concomitant use of spinal and pelvis MRI in patients with suspected SpA and in healthy subjects. One of these (13) demonstrated that the simultaneous evaluation of the spine and the SIJ using MRI can lead to higher diagnostic accuracy. Other authors who have not confirmed this finding (27) sustain that the combined use of spinal and pelvis MRI only moderately increases the diagnostic value in patients with suspected nr-axSpA, because of the inclusion of false positives. In fact, other rheumatic diseases involving the spine such as Scheuermann's disease, spondylodiscitis, erosive osteochondrosis and other degenerative diseases of the intervertebral disc, may show similar patterns on MRI (12). Even the appearance of multiple structural lesions (at least three), just as fatty lesions, increases the probability of axSpA (28), although, according to other studies, the prevalence of these forms of lesions tends to increase with aging and can be present even in healthy individuals or patients affected with other spinal degenerative diseases. Patients with nonspecific LBP and healthy subjects may have some signs suggestive of $\mathrm{SpA}$ such as fatty lesions on spinal MRIs $(12,28)$. According to Weber and colleagues (27) using a MRI of the SIJ alone is less sensitive but more specific than the combined use of the spinal and pelvis MRI, while inclusion of a spinal MRI leads to increased sensitivity and reduced specificity.

The cost and time necessary to carry out MRIs cannot, in any case, be ignored. Nine of the patients (15\%) with inflammatory lesions on spinal MRIs showed no abnormalities on SIJ MRIs, while $12(20 \%)$ patients with sacroiliitis on MRI did not present lesions on spinal MRIs. Our data would seem to indicate that the use of spinal MRIs together with SIJ MRIs can add additional, relevant information during both the diagnostic process and the therapeutic follow-up. The current study investigated if there were any differences in the clinical indices of disease activity commonly used in clinical practice in relation to the presence or absence of signs 
of sacroiliitis on plain radiographs and on MRIs. Although a significant difference was found in the three cohorts with regard to the prevalence of sacroiliitis on MRIs and X-rays and on the SPARCC SIJ score, we did not find any differences in clinical and disease activity indices. Higher indices were not found in the patients with active sacroiliitis on MRI with respect to those without inflammatory changes in the SIJ or with initial signs of radiographic sacroiliitis. This result may depend on both the early stages of axSpA and the small sample size. In fact, several studies (29-34) have reported higher values of clinical, functional and disease activity indices in $\mathrm{r}-\mathrm{axSpA}$, in patients with disease duration of several years with respect to subjects with nr-axSpA. Future studies could examine if there is any correlation between the levels of these indices and the presence/absence of active sacroiliitis in large patient cohorts.

\section{CONCLUSIONS}

It has become increasingly urgent to detect axSpA in its earliest stages in order to initiate treatment as early as possible. MRI can detect inflammatory lesions and signs of active disease process even before structural bone damage has occurred. Pelvis MRI has recently been included in the ASAS classification criteria whose positivity is defined on the basis of the exclusive presence of inflammatory signs of SIJ. It remains a matter of debate whether the inclusion of inflammatory and structural lesions of spinal MRIs in the ASAS classification criteria could help to identify patients with suspected axSpA. A high prevalence of inflammatory lesions on MRIs of the SJI and of the spine (anterior osteitis and enthesitis) were found in our patients. As inflammatory lesions on spinal MRIs can occur in the absence of SIJ involvement, the use of spinal MRIs together with SIJ MRIs may add additional, relevant information to the diagnostic process, especially with regard to nr-axSpA patients without signs of sacroiliitis on MRI. A standard radiograph of the pelvis continues to be a crucial step in the diagnostic investigation in patients with suspected axSpA, especially in those with a longer history of symptoms. Studies on the involvement of the thoracic spine, which has until now never been considered by methods scoring spinal structural damage, are warranted. A significant involvement of the thoracic region was noted in the patients studied.

Conflict of interest: the authors declare no potential conflict of interest.

Contributions: ML, manuscript drafting, data analyzing, acquiring and interpreting; RR and LP, study conceiving and design, data processing and manuscript drafting. ST and CLC, performing of spine and pelvis X-rays and MRI, images reading; FO, statistical analysis, data analyzing and interpreting. PF and AO, data acquiring. All the authors made substantive intellectual contributions to the study, reviewed the article, and gave the final approval of the version being submitted.

Acknowledgments: the authors would also like to thank Linda Inverso for her assistance in editing the English version.

\section{REFERENCES}

1. Rudwaleit M, van der Heijde D, Landewé $\mathrm{R}$, et al. The development of Assessment of SpondyloArthritis international Society classification criteria for axial spondyloarthritis (part II): validation and final selection. Ann Rheum Dis. 2009; 68: 777-83.

2. Rudwaleit M. New approaches to diagnosis and classification of axial and peripheral spondyloarthritis. Curr Opin Rheumatol. 2010; 22: 375-80.

3. Braun J, van den Berg R, Baraliakos X, et al. 2010 update of the ASAS/EULAR recommendations for the management of ankylosing spondylitis. Ann Rheum Dis. 2011; 70 : 896-904.

4. Van der Linden S, Valkenburg HA, Cats A. Evaluation of diagnostic criteria for ankylosing spondylitis. A proposal for modification of the New York criteria. Arthritis Rheum. 1984; 27: 361-8.

5. Sieper J, van der Heijde D, Landewé R, et al. New criteria for inflammatory back pain in patients with chronic back pain: a real pa- 
tient exercise by experts from the Assessment of SpondyloArthritis international Society (ASAS). Ann Rheum Dis. 2009; 68: 784-8.

6. Weber U, Lambert RG, Ostergaard M, et al. The diagnostic utility of magnetic resonance imaging in spondylarthritis: an international multicenter evaluation of one hundred eighty-seven subjects. Arthritis Rheum. 2010; 62: 3048-58.

7. Baraliakos X, van der Heijde D, Braun J, Landewé RB. OMERACT magnetic resonance imaging initiative on structural and inflammatory lesions in ankylosing spondylitis-report of a special interest group at OMERACT 10 on sacroiliac joint and spine lesions. J Rheumatol. 2011; 38: 2051-4.

8. Rudwaleit M, Jurik AG, Hermann KG, et al. Defining active sacroiliitis on magnetic resonance imaging (MRI) for classification of axial spondyloarthritis: a consensual approach by the ASAS/OMERACT MRI group. Ann Rheum Dis. 2009; 68: 1520-7.

9. Spadaro A, Lubrano E, Marchesoni A, et al. The adherence to ASAS classification criteria and to ASAS recommendations for the use of anti-TNF-alpha agents in axial spondyloarthritis. Clin Exp Rheumatol. 2014; 32: 465-70.

10. Spadaro A, Lubrano E, Marchesoni A, et al. Remission in ankylosing spondylitis treated with anti-TNF- $\alpha$ drugs: a national multicentre study. Rheumatology (Oxford). 2013; 52: 1914-9.

11. Lubrano E, Perrotta FM, Marchesoni A, et al. Remission in non radiographic axial spondyloarthritis treated with anti-tumor necrosis factor- $\alpha$ drugs: an Italian multicenter study. J Rheumatol. 2015; 42: 258-63.

12. Hermann KG, Baraliakos X, van der Heijde DM, et al. Descriptions of spinal MRI lesions and definition of a positive MRI of the spine in axial spondyloarthritis: a consensual approach by the ASAS/OMERACT MRI study group. Ann Rheum Dis. 2012; 71: 1278-88.

13. van der Heijde D, Sieper J, Maksymowych WP, et al. Spinal inflammation in the absence of sacroiliac joint inflammation on magnetic resonance imaging in patients with active non radiographic axial spondyloarthritis. Arthritis Rheumatol. 2014; 66: 667-73.

14. Braun J, Baraliakos X, Golder W, et al. Magnetic resonance imaging examinations of the spine in patients with ankylosing spondylitis, before and after successful therapy with infliximab: evaluation of a new scoring system. Arthritis Rheum. 2003; 48:1126-36.

15. Mackay K, Brophy S, Mack C, et al. The development and validation of a radiographic grading system for the hip in ankylosing spondylitis: the bath ankylosing spondylitis radiology hip index. J Rheumatol. 2000; 27 : 2866-72.
16. Creemers MC, Franssen MJ, van't Hof MA, et al. Assessment of outcome in ankylosing spondylitis: an extended radiographic scoring system. Ann Rheum Dis. 2005; 64: 127-9.

17. Salaffi F, Carotti M, Garofalo G, et al. Radiological scoring methods for ankylosing spondylitis: a comparison between the bath ankylosing spondylitis radiology index and the modified stoke ankylosing spondylitis spine score. Clin Exp Rheumatol. 2007; 25: 67-74.

18. Maksymowych WP, Inman RD, Salonen D, et al. Spondyloarthritis research consortium of Canada magnetic resonance imaging index for assessment of sacroiliac joint inflammation in ankylosing spondylitis. Arthritis Rheum. 2005; 53: 703-9.

19. Maksymowych WP, Inman RD, Salonen D, et al. Spondyloarthritis research consortium of Canada magnetic resonance imaging index for assessment of spinal inflammation in ankylosing spondylitis. Arthritis Rheum. 2005; 53: 502-9.

20. de Hooge M, van den Berg R, NavarroCompán V, et al. Magnetic resonance imaging of the sacroiliac joints in the early detection of spondyloarthritis: no added value of gadolinium compared with short tau inversion recovery sequence. Rheumatology (Oxford). 2013; 52: 1220-4.

21. van den Berg R, de Hooge M, Bakker PA, et al. Metric properties of the SPARCC score of the sacroiliac joints - data from baseline, 3-month, and 12-month follow up in the SPACE cohort. J Rheumatol. 2015; 42: 1186-93.

22. Lukas C, Braun J, van der Heijde D, et al. Scoring inflammatory activity of the spine by magnetic resonance imaging in ankylosing spondylitis: a multireader experiment. J Rheumatol. 2007; 34: 862-70.

23. Bennett AN, Rehman A, Hensor EM, et al. Evaluation of the diagnostic utility of spinal magnetic resonance imaging in axial spondylarthritis. Arthritis Rheum. 2009; 60:1331-41.

24. Weber U, Hodler J, Kubik RA, et al. Sensitivity and specificity of spinal inflammatory lesions assessed by whole-body magnetic resonance imaging in patients with ankylosing spondylitis or recent-onset inflammatory back pain. Arthritis Rheum. 2009; 61: 900-8.

25. de Hooge $M$, van den Berg R, NavarroCompán V, et al. Patients with chronic back pain of short duration from the SPACE cohort: which MRI structural lesions in the sacroiliac joints and inflammatory and structural lesions in the spine are most specific for axial spondyloarthritis? Ann Rheum Dis. 2016; 75 : 1308-14.

26. Baraliakos X, Landewé R, Hermann KG, et al. Inflammation in ankylosing spondylitis: a systematic description of the extent and frequency of acute spinal changes using mag- 
netic resonance imaging. Ann Rheum Dis. 2005; 64: 730-4.

27. Weber U, Zubler V, Zhao Z, et al. Does spinal MRI add incremental diagnostic value to MRI of the sacroiliac joints alone in patients with non-radiographic axial spondyloarthritis? Ann Rheum Dis. 2015; 74: 985-92.

28. Song IH, Hermann KG, Haibel H, et al. Relationship between active inflammatory lesions in the spine and sacroiliac joints and new development of chronic lesions on whole-body MRI in early axial spondyloarthritis: results of the ESTHER trial at week 48. Ann Rheum Dis. 2011; 70: 1257-63.

29. Robinson PC, Benham H. Advances in classification, basic mechanisms and clinical science in ankylosing spondylitis and axial spondyloarthritis. Intern Med J. 2015; 45: 127-33.

30. Pedersen SJ, Sørensen IJ, Garnero P, et al. ASDAS, BASDAI and different treatment responses and their relation to biomarkers of inflammation, cartilage and bone turnover in patients with axial spondyloarthritis treated with TNFa inhibitors. Ann Rheum Dis. 2011; 70: 1375-81.
31. Pedersen SJ, Sørensen IJ, Lambert RG, et al. Radiographic progression is associated with resolution of systemic inflammation in patients with axial spondylarthritis treated with tumor necrosis factor $\alpha$ inhibitors: a study of radiographic progression, inflammation on magnetic resonance imaging, and circulating biomarkers of inflammation, angiogenesis, and cartilage and bone turnover. Arthritis Rheum. 2011; 63: 3789-800.

32. Poddubnyy DA, Rudwaleit M, Listing J, et al. Comparison of a high sensitivity and standard $\mathrm{C}$ reactive protein measurement in patients with ankylosing spondylitis and nonradiographic axial spondyloarthritis. Ann Rheum Dis. 2010; 69: 1338-41.

33. Visvanathan S, Wagner C, Marini JC, et al. Inflammatory biomarkers, disease activity and spinal disease measures in patients with ankylosing spondylitis after treatment with infliximab. Ann Rheum Dis. 2008; 67: 511-7.

34. Reveille JD. Biomarkers for diagnosis, monitoring of progression, and treatment responses in ankylosing spondylitis and axial spondyloarthritis. Clin Rheumatol. 2015; 34: 1009-18. 